\title{
Supplemental information for: Postsynthetic and Selective Control of Lead Halide Perovskite Microlasers
}

Nan Zhang ${ }^{\dagger, \perp}$, Kaiyang Wang ${ }^{\dagger, \perp}$, Haohan $\mathrm{Wei}^{\dagger}$, Zhiyuan $\mathrm{Gu}^{\dagger}$, Wenzhao Sun ${ }^{\dagger}$, Jiankai $\mathrm{Li}^{\dagger}$, Shumin Xiao ${ }^{\ddagger *}$, and Qinghai Song ${ }^{\dagger *}$

†National Key Laboratory on Tunable Laser Technology, Department of Electrical and Information Engineering, Harbin Institute of Technology, Shenzhen, 518055, China

${ }^{\ddagger}$ Department of Material Science and Engineering, Harbin Institute of Technology, Shenzhen, 518055, China.

${ }^{\perp}$ These authors contributed equally to this work

Corresponding to: qinghai.song@hitsz.edu.cn; shumin.xiao@hitsz.edu.cn

I. Absorption of the $\mathrm{CH}_{3} \mathrm{NH}_{3} \mathrm{PbBr}_{3}$ microwire and microplate

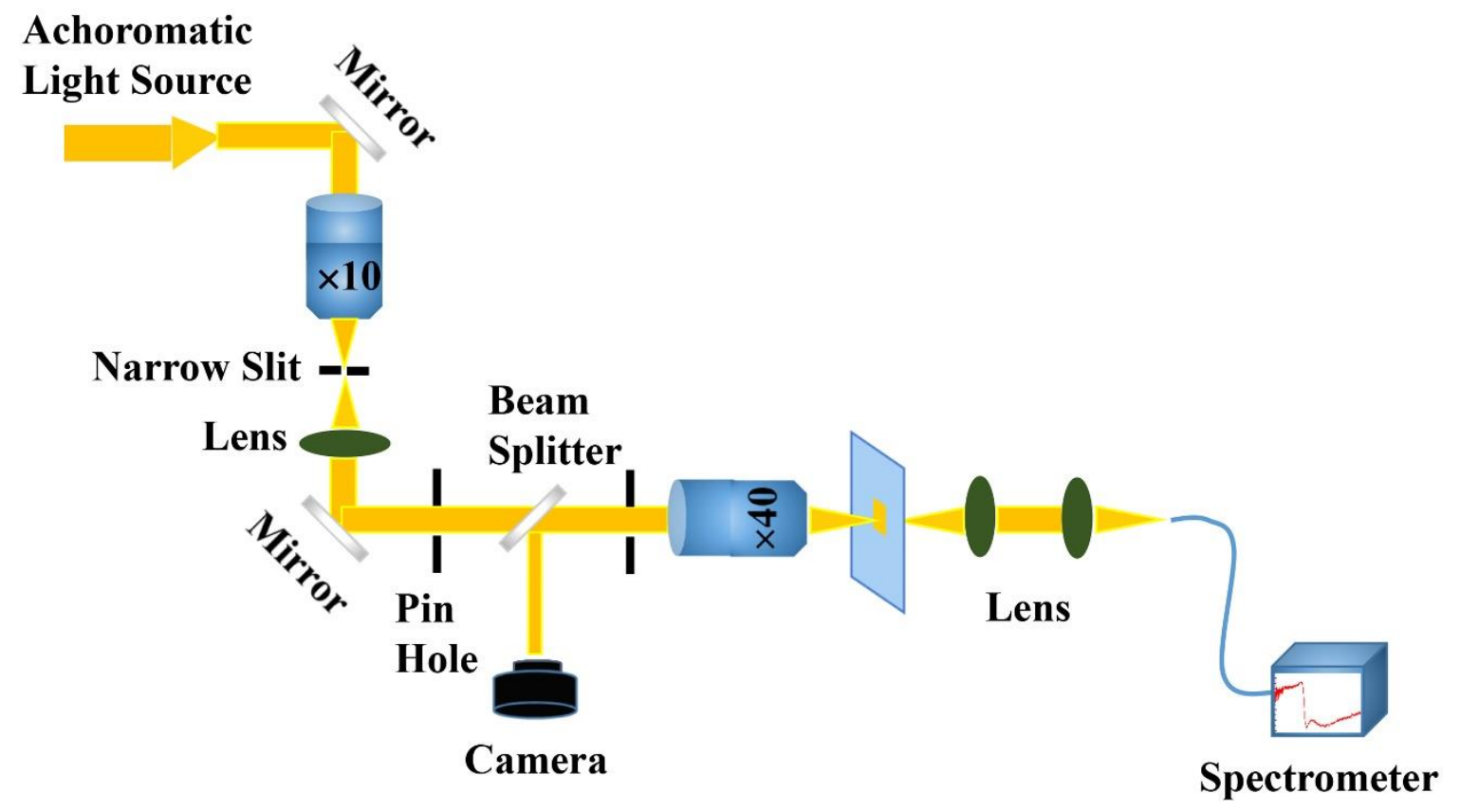


Figure S1. Optical setup for measuring the absorption spectrum of single microplate and microwire.

II. Characterization of the photoluminescence from $\mathrm{CH}_{3} \mathrm{NH}_{3} \mathrm{PbBr}_{3}$ microwire

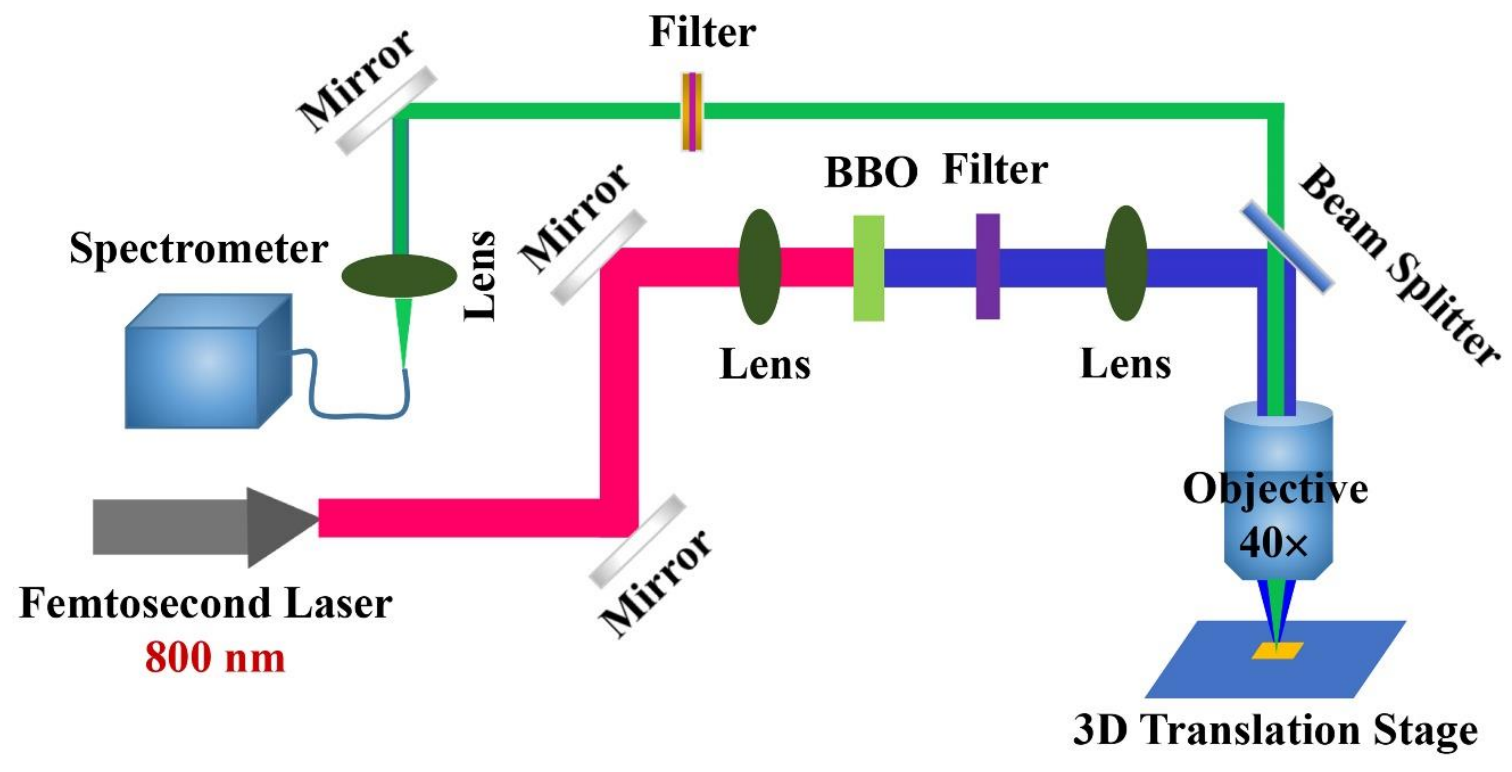

Figure S2. The setup for measuring the photoluminescence and laser emissions from the lead halide perovskite. 


\section{Perovskite microplate size as a function of reaction time}

(a)

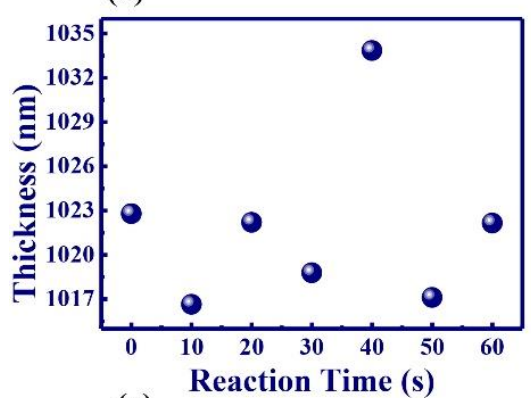

(c)

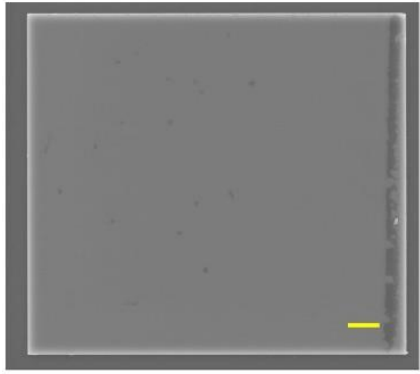

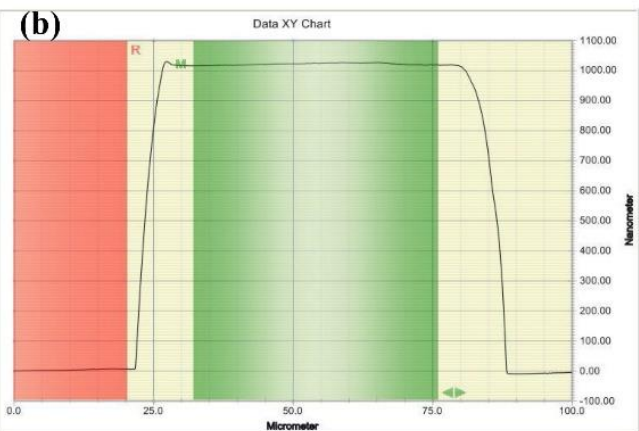

(d)

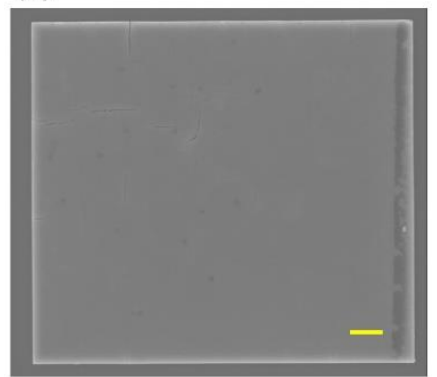

Figure S3. (a) The perovskite microplate thickness as a function of reaction time in the ICP chamber. (b) Profilometer measurement result of the original microplate. (c) and (d) are the scanning electron microscope (SEM) images of another perovskite microplate before and after reaction in ICP for $60 \mathrm{sec}$, where the scale bar is $2 \mu \mathrm{m}$.

\section{The photoluminescence of $\mathrm{CH}_{3} \mathrm{NH}_{3} \mathrm{PbBr}_{3}$ microwire by reaction with chlorine gas}

In the main text, we have done a control experiment to process the lead halide perovskite with chlorine gas. Here we show the detail experimental results. Figure S4(a) shows the top-view scanning electron microscope (SEM) image of the $\mathrm{CH}_{3} \mathrm{NH}_{3} \mathrm{PbBr}_{3}$ microplate. The sizes of this microplate are quite similar to the one in Figure 2. As shown in Figure S4(b), the energy dispersive X-ray spectroscopy (EDS) analysis of this perovskite microplate processed with chlorine gas can show this difference obviously. After reaction with chlorine gas, the percentage of $\mathrm{Cl}^{-}$in perovskite is negligibly small.

When the pumping power is $3.6 \mu \mathrm{J} / \mathrm{cm}^{2}$, the recorded PL spectrum is shown as the black solid line in Figure S4(c). It is a broad peak centered at $537 \mathrm{~nm}$ with full width at half maximum (FWHM) around $30 \mathrm{~nm}$. After reaction in chlorine gas for 30 seconds, the 
PL spectrum is shown as the red dash line in Figure S4(c). We can see that the peak intensity decreases around $40 \%$ and a second tiny peak appears at $511 \mathrm{~nm}$. Compared with processing in $\mathrm{Cl}^{-}$, the $\mathrm{PL}$ at shorter wavelength is more than $50 \%$ smaller. This difference is more dramatic in laser spectrum, when the pumping power is $4.2 \mu \mathrm{J} / \mathrm{cm}^{2}$, the laser spectrum of the as-grown microplate is shown as the black solid line in Figure S4(d). The laser peak centered at $552 \mathrm{~nm}$. After processing in chlorine gas for 30 seconds, there is no laser any more. Instead, there is a broad peak centered at $533 \mathrm{~nm}$ with full width at half maximum (FWHM) around $30 \mathrm{~nm}$.

(a)

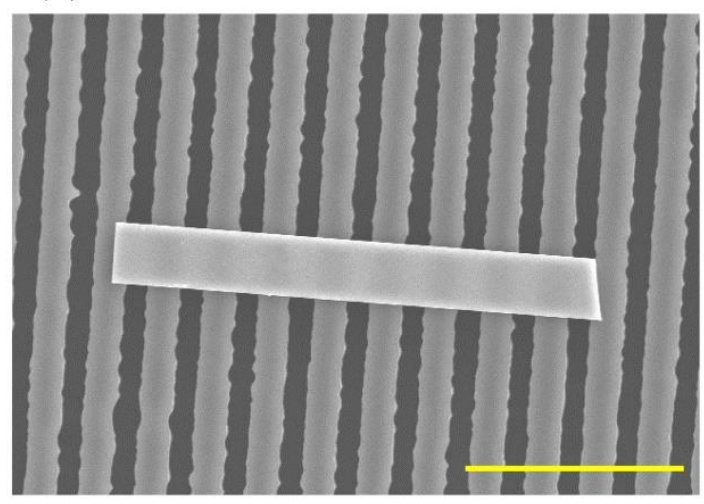

(c)

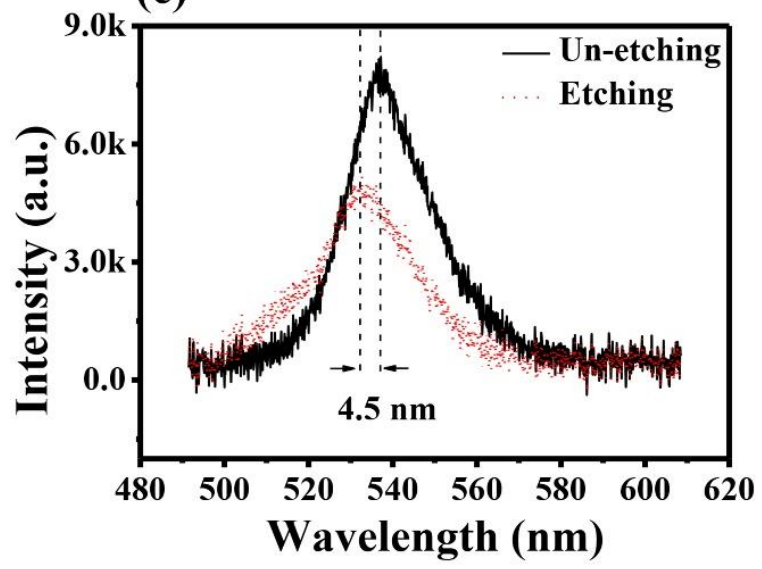

(b)

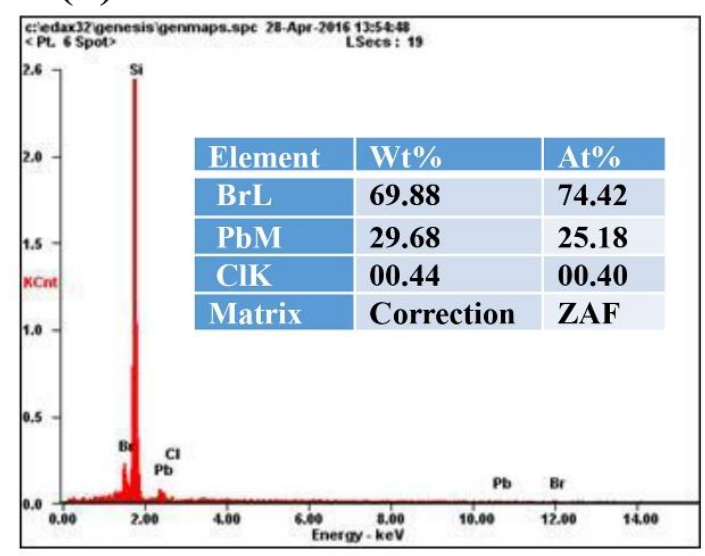

(d)

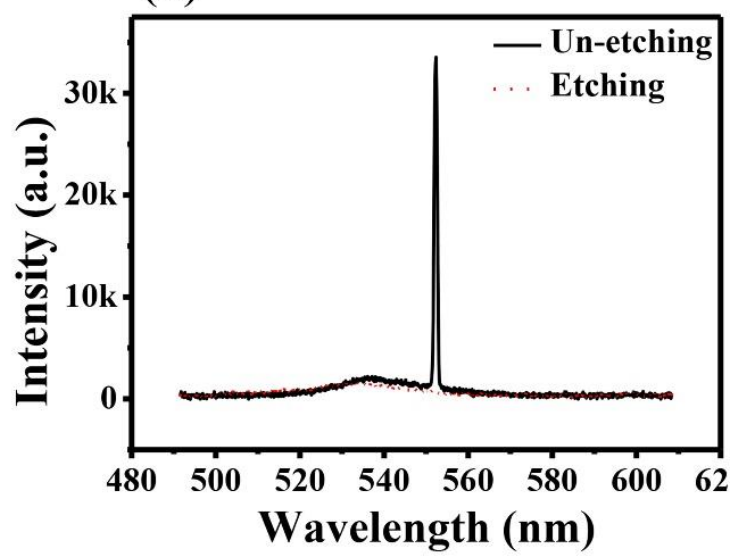

Figure S4. (a) The top-view SEM image of the $\mathrm{CH}_{3} \mathrm{NH}_{3} \mathrm{PbBr}_{3}$ microplate, where the scale bar is $20 \mu \mathrm{m}$. (b) Energy-dispersive X-ray spectroscopy (EDS) of the microplate. (c) The PL spectrum before and after the reaction with chlorine gas. (d) The laser spectrum before and after the reaction with chlorine gas. 


\section{The reaction of $\mathrm{CH}_{3} \mathrm{NH}_{3} \mathrm{PbBr}_{3}$ microplate with higher ICP power}

In the main text, we have done a series of experiments to process the lead halide perovskite with ICP power of $400 \mathrm{~W}$. Here we show the influences of ICP power. On example is shown in Figure S5, which was recorded by increasing ICP power to 500W. All the other parameters are the same as Figure 2. Figure S5(a) shows the top-view SEM image of the $\mathrm{CH}_{3} \mathrm{NH}_{3} \mathrm{PbBr}_{3}$ microplate, and in Figure $\mathrm{S} 5(\mathrm{~b})$ we show a partial enlarged view of the microplate. We can see that the $\mathrm{CH}_{3} \mathrm{NH}_{3} \mathrm{PbBr}_{3}$ microplate has been damaged seriously. Through the corresponding fluorescent microscope image shown in the Figure 5(c), it is more intuitive to see this damage. When the pumping density is above 8.6 $\mu \mathrm{J} / \mathrm{cm}^{2}$, filtered the pump laser, we cannot see any emitted light. By compared the absorption curves before and after reaction (in Figure S5(d)), this damage was demonstrated once again. So that, the higher ICP power will not only damaged the structure but also can eliminate the luminescent properties of the $\mathrm{CH}_{3} \mathrm{NH}_{3} \mathrm{PbBr}_{3}$ microplate. 
(a)

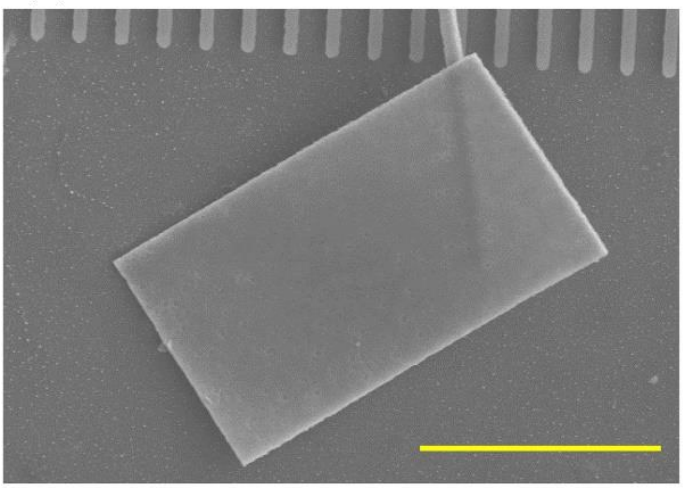

(c)

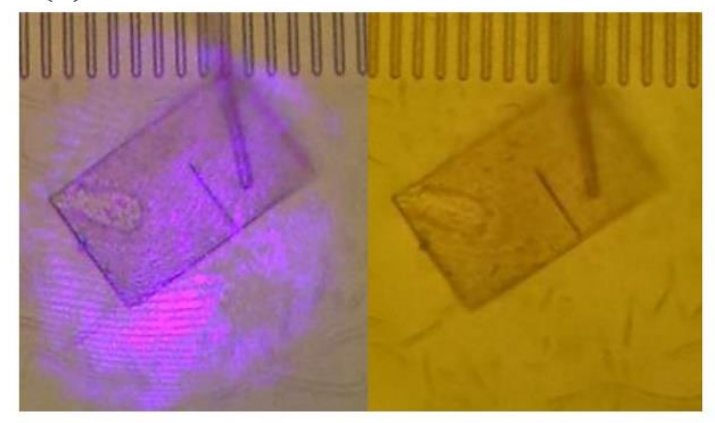

(b)

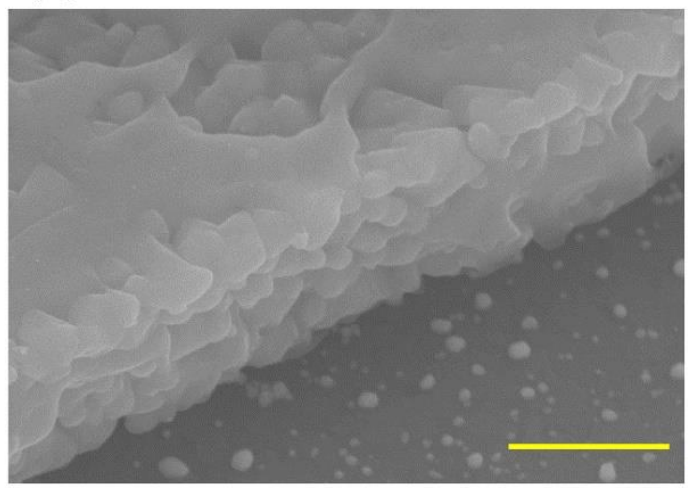

(d)

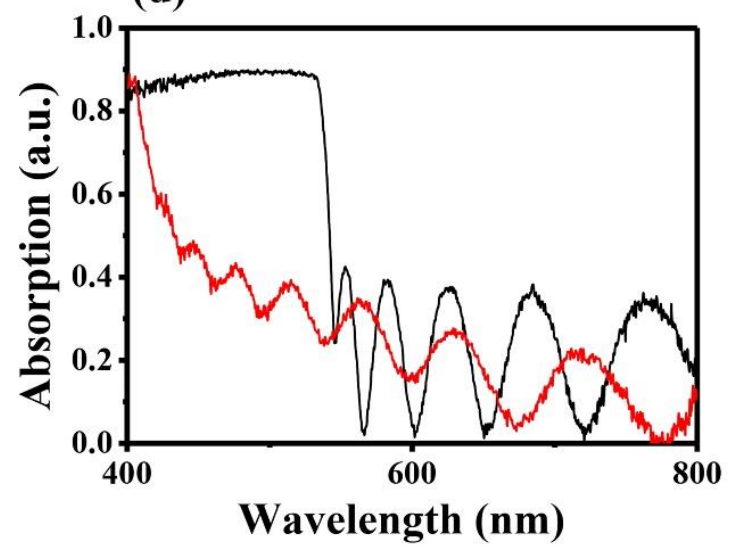

Figure S5. The top-view (a) and side-view (b) SEM image of the $\mathrm{CH}_{3} \mathrm{NH}_{3} \mathrm{PbBr}_{3}$ microplate with 500W ICP power where the scale bar are $30 \mu \mathrm{m}$ and $1 \mu \mathrm{m}$, respectively.

(c) The fluorescent microscope images with and without pump light filter of sample in (a). (d) The absorption spectra before (black line) and after (red line) ICP processing with $500 \mathrm{~W}$ ICP power.

\section{The lasing actions in $\mathrm{CH}_{3} \mathrm{NH}_{3} \mathrm{PbBr}_{3}$ microplate with different reaction time}

We have also studied the lasing actions in lead halide perovskite microplate numerically. The numerical calculation is based on a commercial finite element method package (Comsol Multiphysics 3.5a). As the measured lasers are all TM polarized (E is in plane), we only consider the TM resonances in numerical calculation. The width and thickness of the perovskite microplate are $7.22 \mu \mathrm{m}$ and $853 \mathrm{~nm}$, respectively. The calculated results are shown in Figure S6. Before ICP processing, we can see a number of 
high Q resonances within the wavelength range from $550 \mathrm{~nm}$ to $560 \mathrm{~nm}$. Around the lasing wavelength in experiment, a high Q mode (Mode-1) has been observed. The Q factor is around 1100, which is far above the other modes and is consistent with the single mode operation in real experiment. Figure S6(c) shows the corresponding field pattern. We can see that is a simple whispering gallery mode.

Figure S6(b) shows the calculated resonant modes in ICP processed perovskite microplate. After the reaction in ICP for 60 seconds, we know from the main text that both the lasing wavelengths and refractive index are changed. Consequently, the lasing actions within the same lead halide perovskite microplate will not be the same. As shown in Figure S6(b), there are two resonances with relatively high Q factors. From their lasing wavelengths, we know that mode- 1 and mode- 2 correspond to the double laser peaks in Figure S6(m). The field pattern of mode-1 is shown in Figure S6(d). We can see that the field pattern is similar to the one in Figure S6(c). We note that the mode spacing is a little bit different from the real experiments. This is caused by the group index of materials. 
(a)

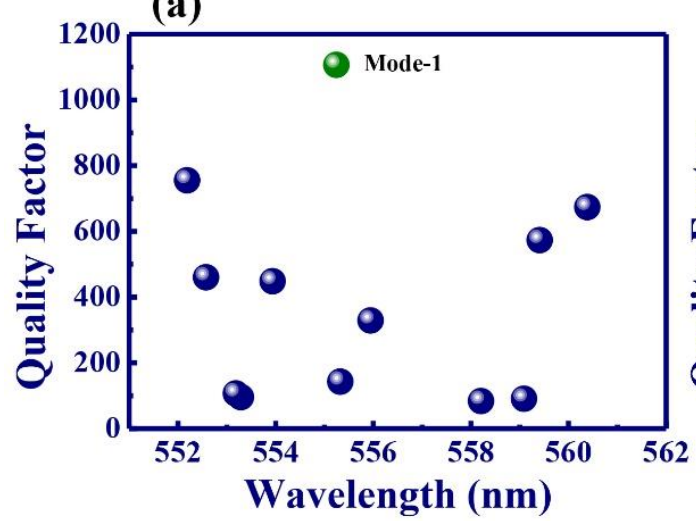

(b)

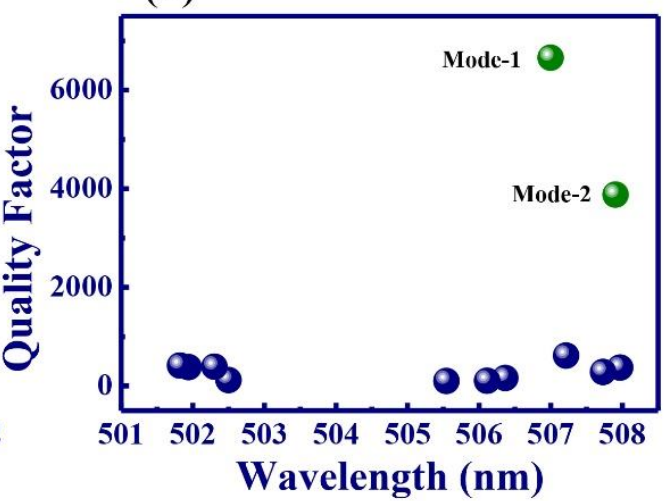

(c)

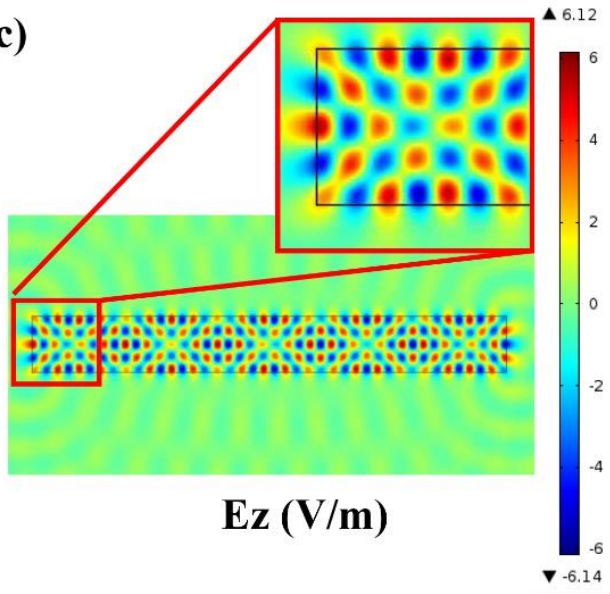

(d)

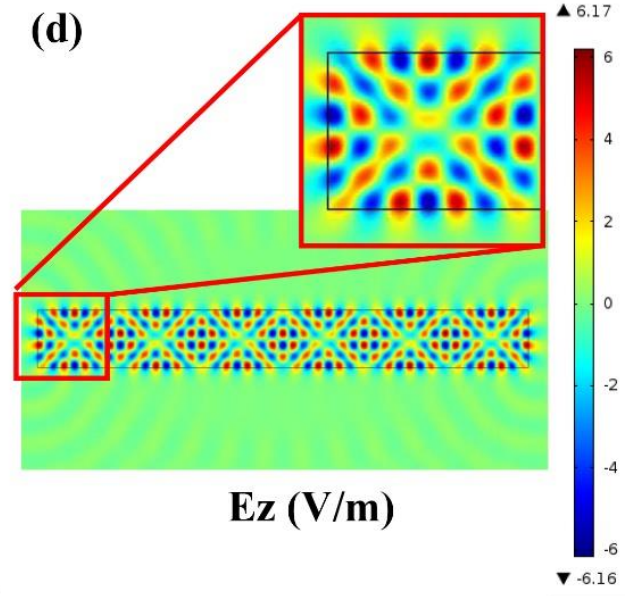

Figure S6. (a) and (b) are the calculated resonances in lead halide perovskite microplates.

(c) and (d) are the corresponding field patterns of mode-1 in (a) and (b), respectively.

In the laser experiment, with the increase of reaction time, the laser peak exhibits blue shift trend, and the thresholds are well maintained in Figure 3. Here we show the detailed experimental results. The right column images of Figure S7 show the laser threshold and the full width at half maximum (FWHM) curves with different reaction time. We can see that the threshold and the intensity are very close at different reaction time. These phenomena demonstrate that the reaction with chloride doesn't damage the structure and the luminescence properties of $\mathrm{CH}_{3} \mathrm{NH}_{3} \mathrm{PbBr}_{3}$ microplate. The detail spectrum of the microplate with different reaction time are shown in the left column images of Figure S7. 
(a)

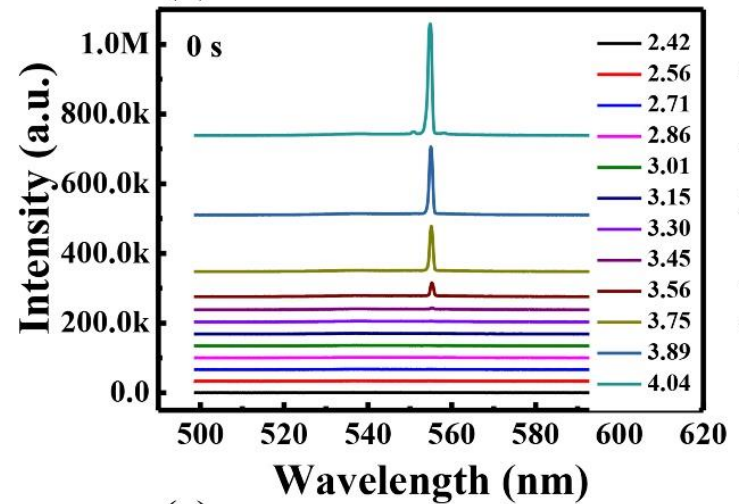

(c)

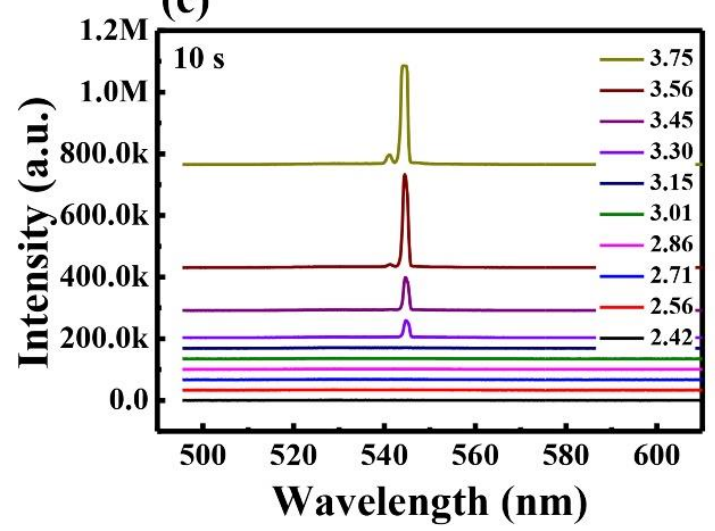

(e)

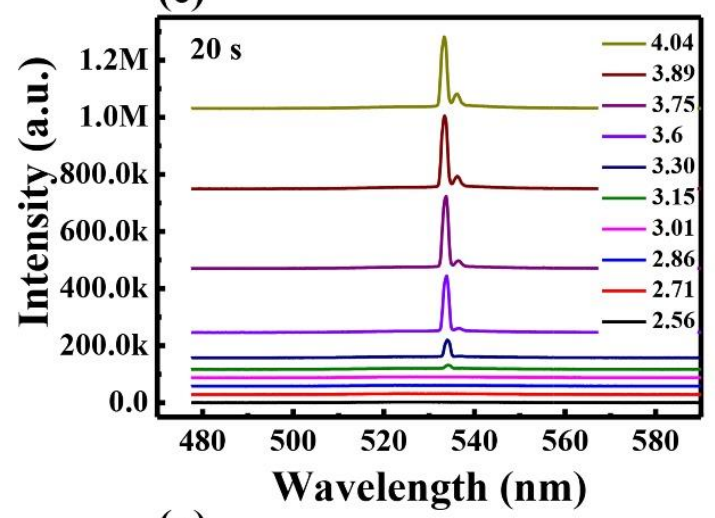

(g)

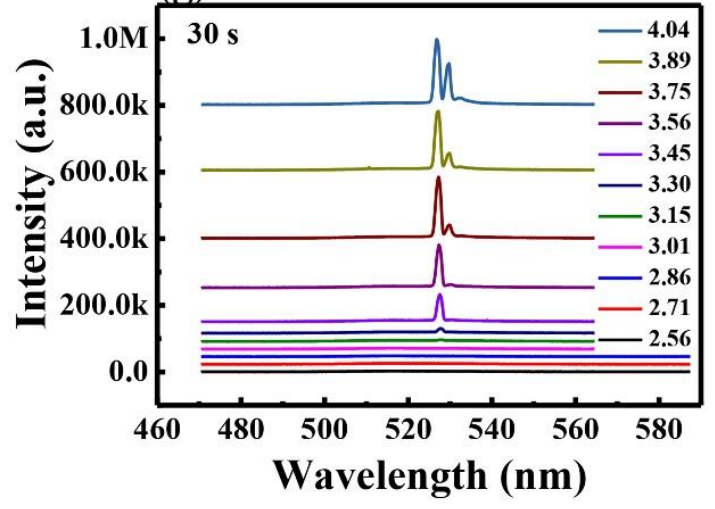

(b)

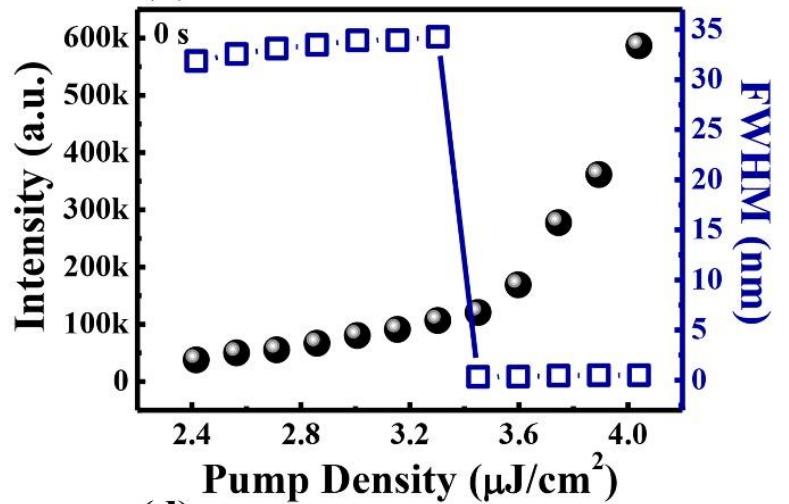

(d)

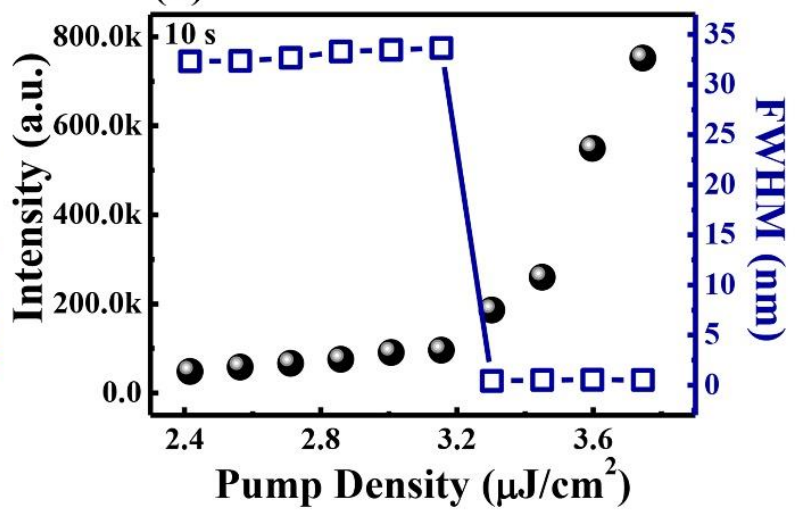

(f)

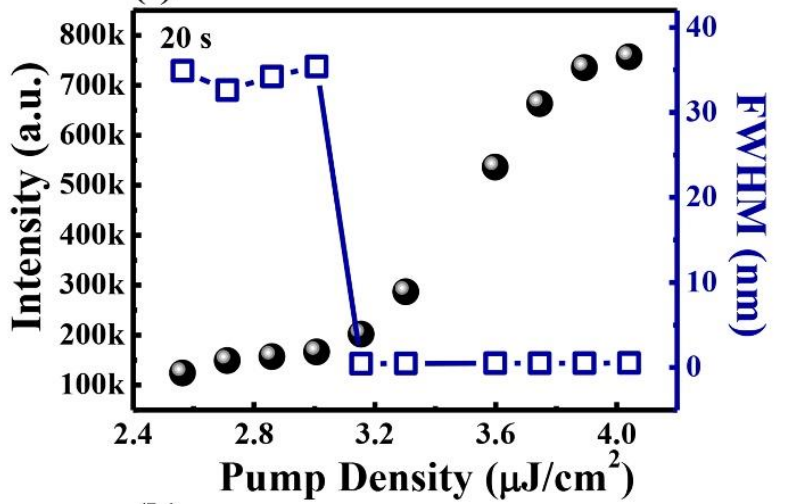

(h)

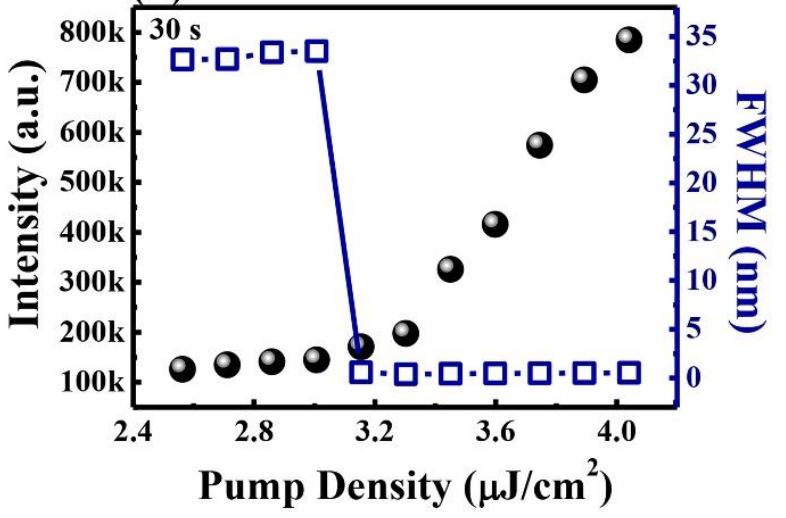


(i)

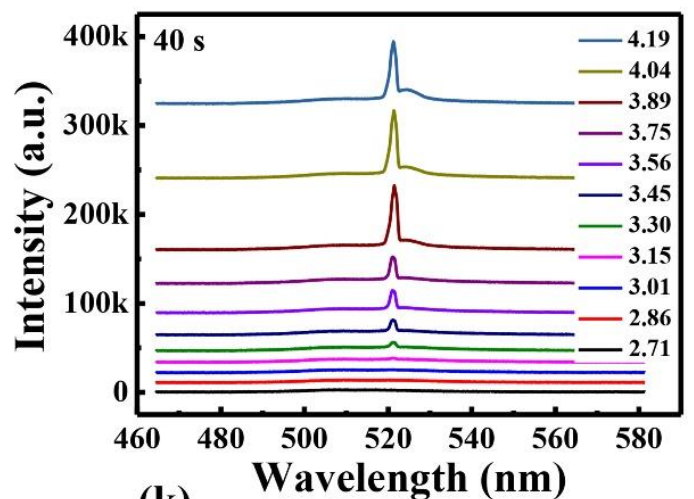

(k)

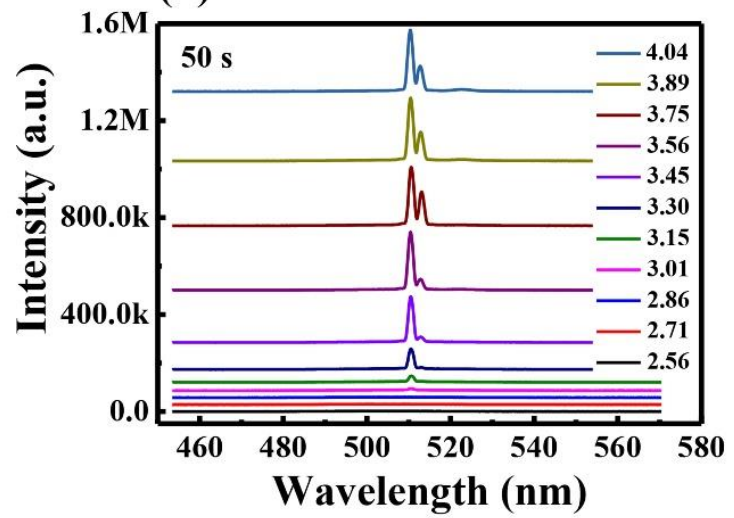

(m)

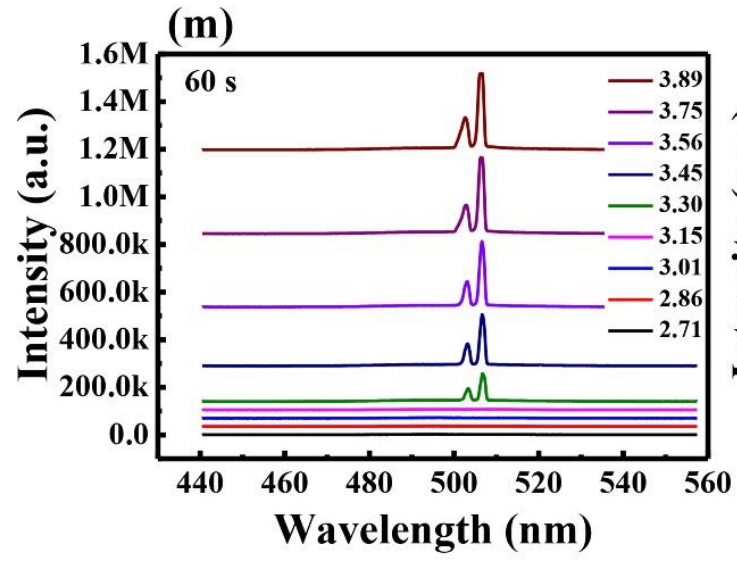

(j)

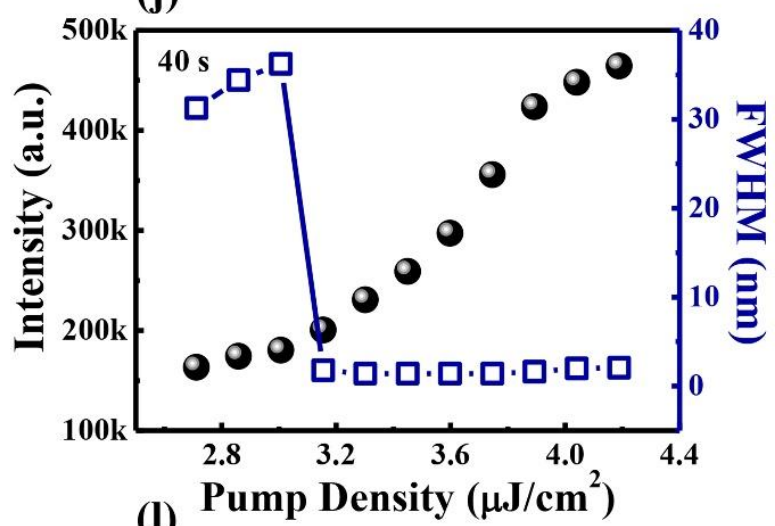

(l)

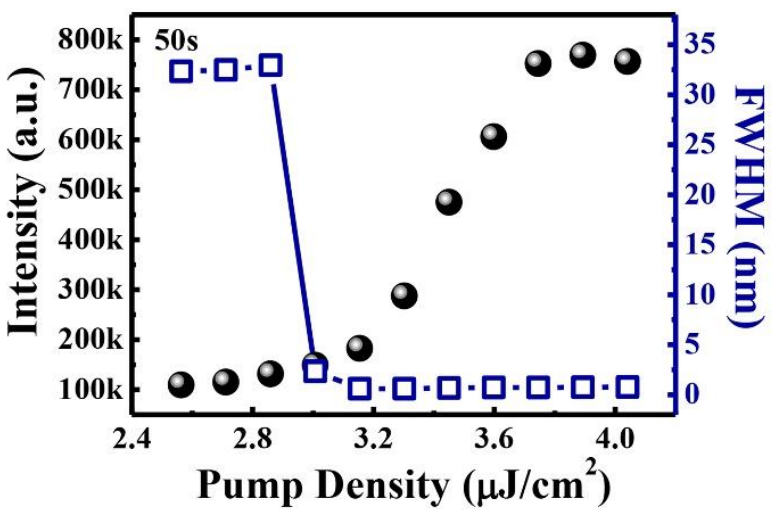

(n)

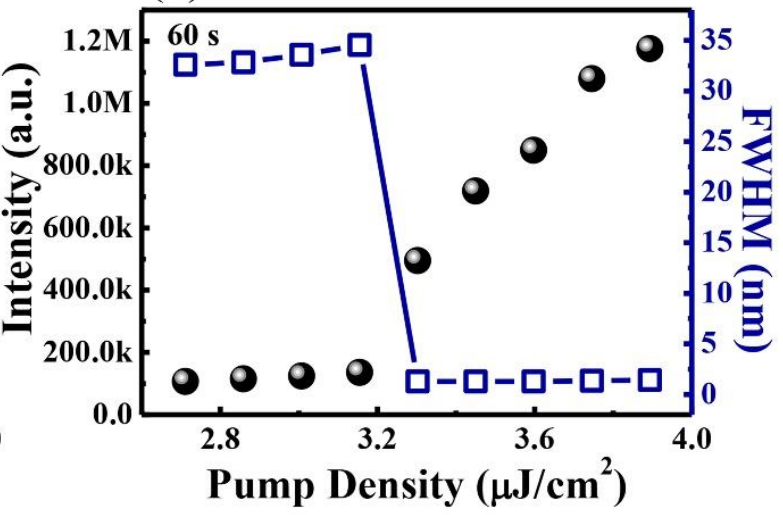

Figure S7. The left column shows the corresponding detailed spectra at different pumping density and the right column shows the threshold and the full width at half maximum (FWHM) curves at different reaction time 0s, 10s, 20s, 30s, 40s, 50s, 60s, respectively..

VII. The lasing actions of microlaser array 
In Figure 4 of the main text, a microlaser array comprising an un-processed microwire (marked by "c") and two ICP processed microwires ("a" with 50s and "b" with 30s, respectively) has been demonstrated. The detailed lasing actions are shown in Figure S8. The lasing wavelength shift induced by reaction in ICP has been shown in Figure S8(a), where the solid line is the un-processed lasing wavelength and the dashed line stands for the processed wavelength. The wavelength shift is proportional to the reaction time, which is consistent with the above analysis. The evolution from spontaneous emission to lasing action is presented in Figure S8(b), where narrow peaks emerge as the pump density is above $4.2 \mu \mathrm{J} / \mathrm{cm}^{2}$. The integrated intensity as a function of pump density is plotted in Figure S8(c), where a lasing threshold can be clearly observed at $4.2 \mu \mathrm{J} / \mathrm{cm}^{2}$, consistent with (b). The polarization characteristics are shown in Figure S8(d), where a transverse electric (TE) polarization is overwhelmingly dominant.

(a)
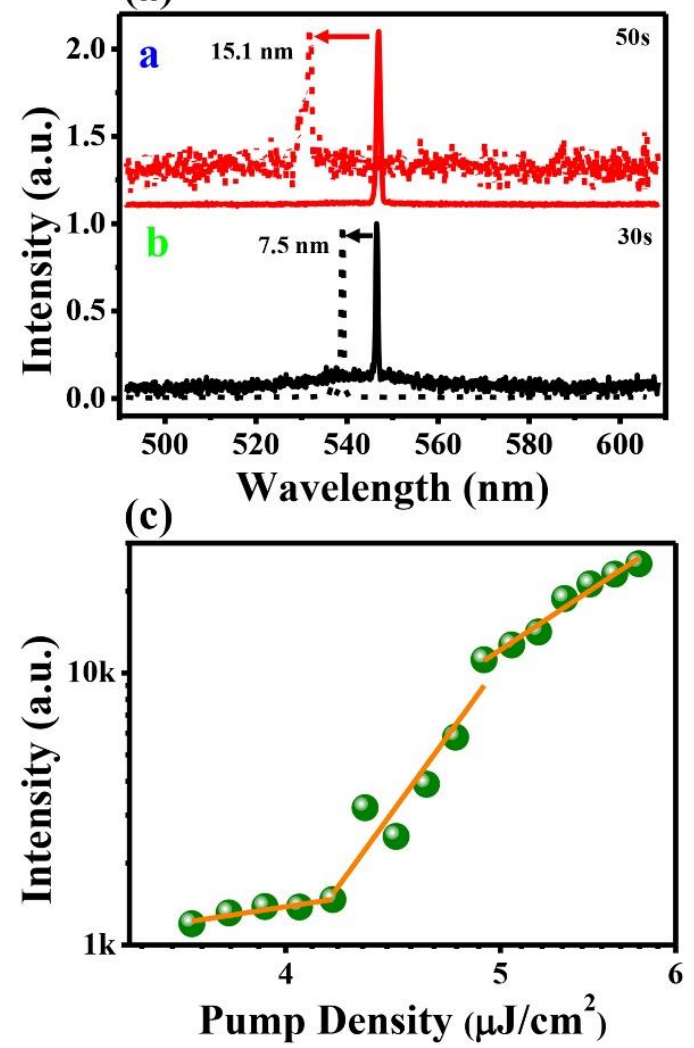

(b)

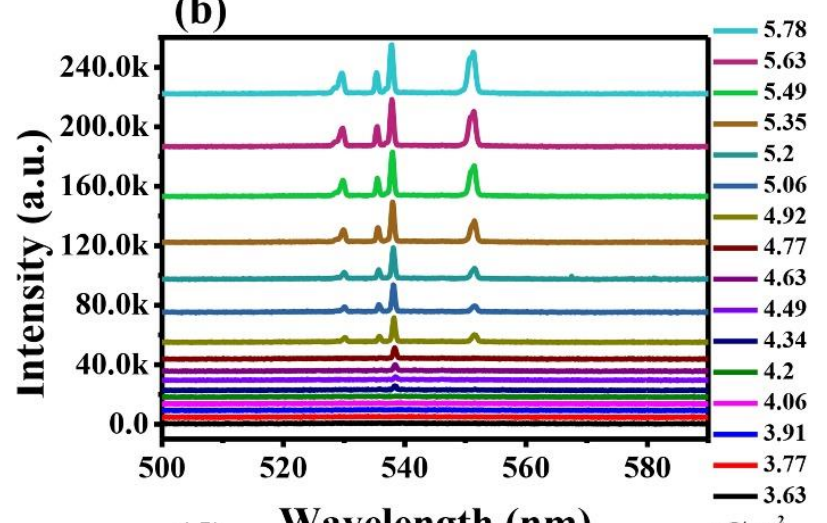

(d)

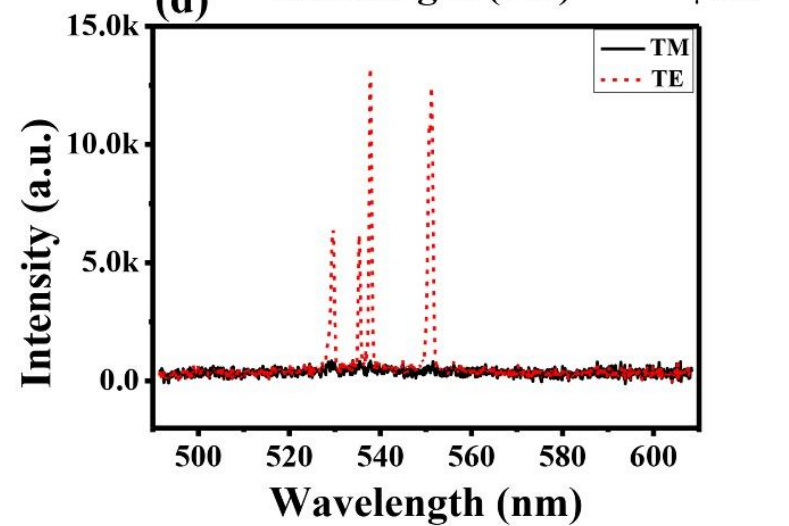

Figure S8. (a) The lasing wavelength shift of microwires under different reaction time in ICP, where the solid line stands for the spectrum of un-processed microwire and the dashed line for processed one. (b) Evolution from spontaneous emission to lasing action 
with the increase of pump density. (c) Log-log plot of integrated intensity as a function of pump density. (d) The polarization of lasing action under pump density of $5.49 \mu \mathrm{J} / \mathrm{cm}^{2}$.

\section{The process for selective control of a single $\mathrm{CH}_{3} \mathrm{NH}_{3} \mathrm{PbBr}_{3}$ microplate and the corresponding lasing actions}

The sample was fabricated by electron beam lithography (EBL). The PMMA photoresist was spin on the $\mathrm{CH} 3 \mathrm{NH} 3 \mathrm{PbBr} 3$ microplate in $2000 \mathrm{rad} / \mathrm{min}$ speed with three times. Through electron beam lithography (EBL) process, the half of the $\mathrm{CH} 3 \mathrm{NH} 3 \mathrm{PbBr} 3$ microplate was covered by the photoresist, and the other half of the microplate was exposed. So that after induced coupled plasma (ICP) process using chloride with 400W ICP power, the exposed half of the $\mathrm{CH}_{3} \mathrm{NH}_{3} \mathrm{PbBr}_{3}$ microplate will be injected into chloride, and the luminous band will be blue shift. The corresponding lasing actions were shown in the Figure S9 and Figure S10. In Figure S9, we can see that when we pumped the half of the covered $\mathrm{CH} 3 \mathrm{NH} 3 \mathrm{PbBr} 3$ microplate, the spectra were centered around $554 \mathrm{~nm}$. When we pumped the other half of the microplate, the spectrum were blue shift at $532 \mathrm{~nm}$. Figure S10 was the threshold and the full width at half maximum (FWHM) curves of the above two pumped situations. 
(a)

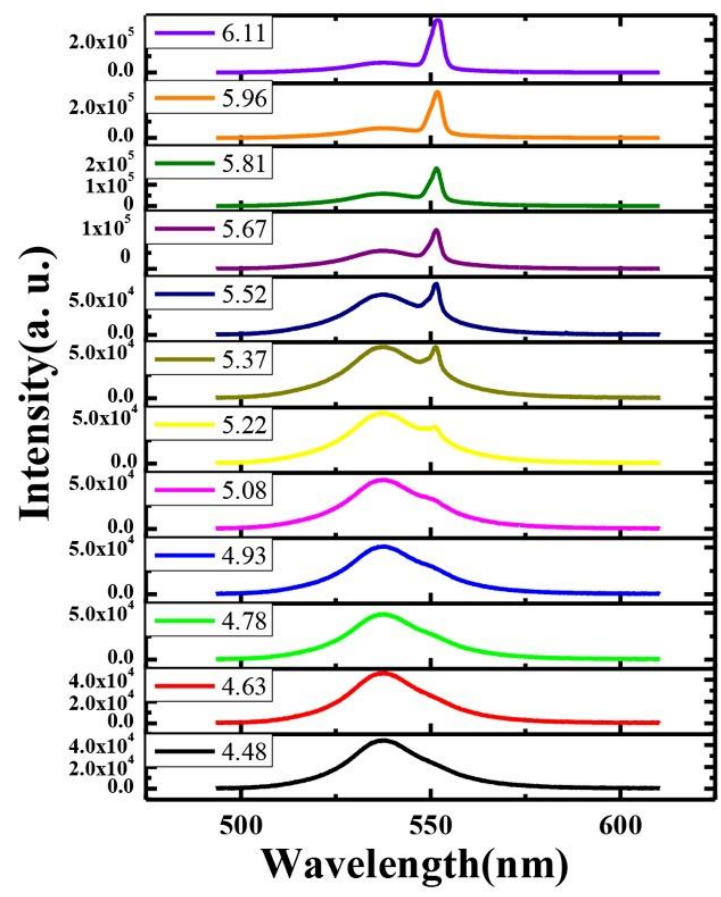

(c)

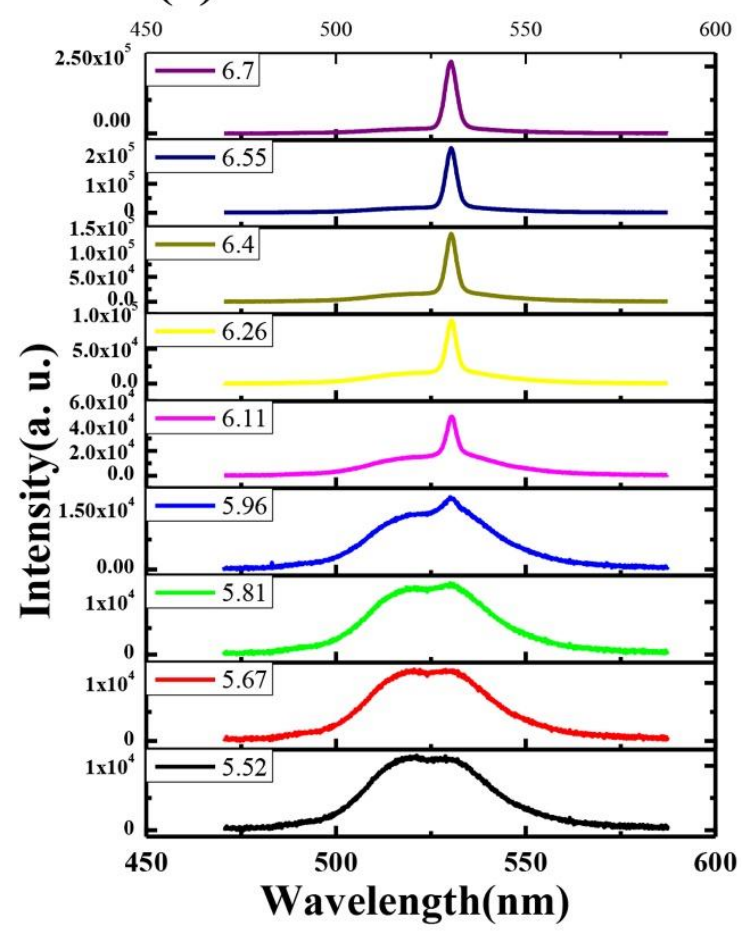

(b)

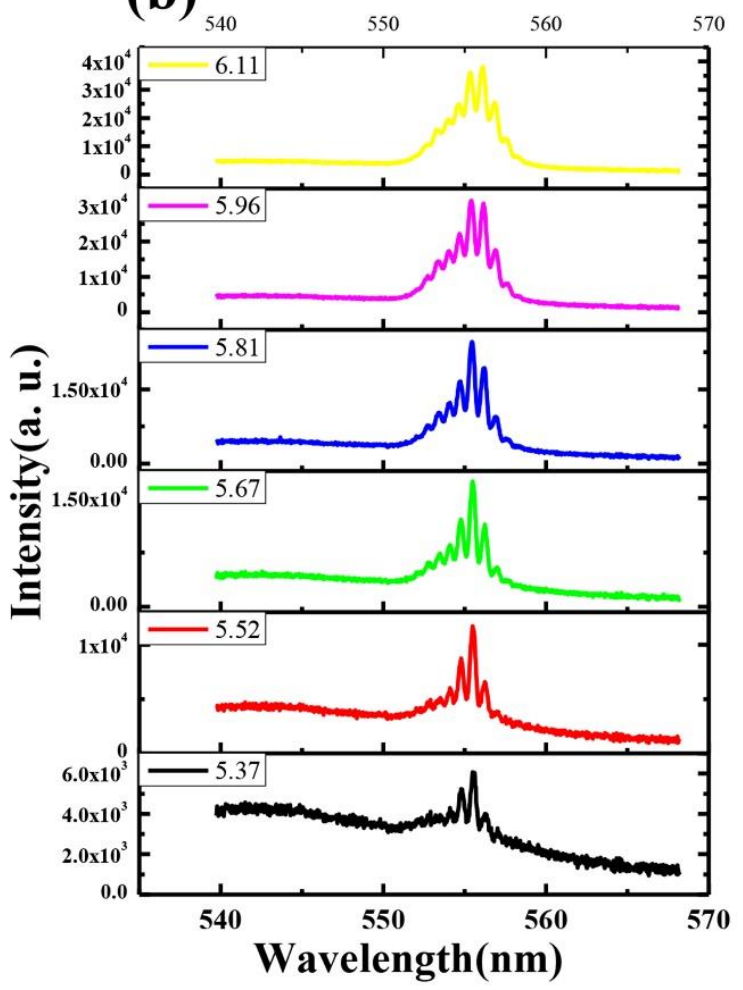

(d)

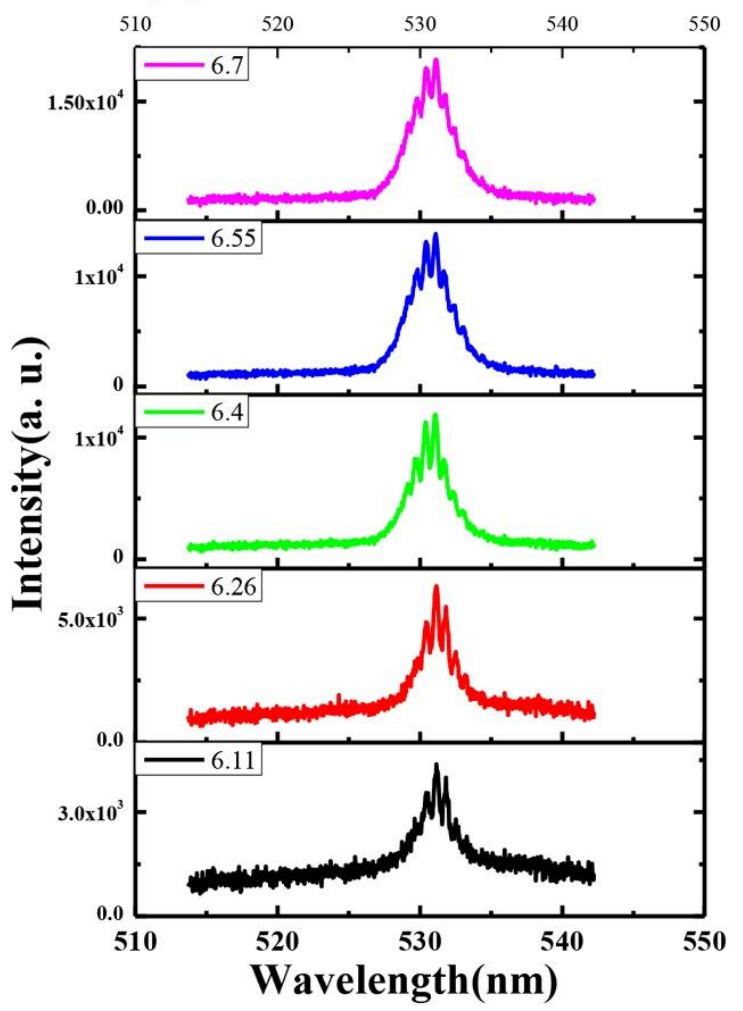


Figure S9. (a), (c) are the spectrum of pumped covered half microplate, exposed half microplate and full pumped, respectively. (b), (d) are their corresponding high-resolution spectrum.

(a)

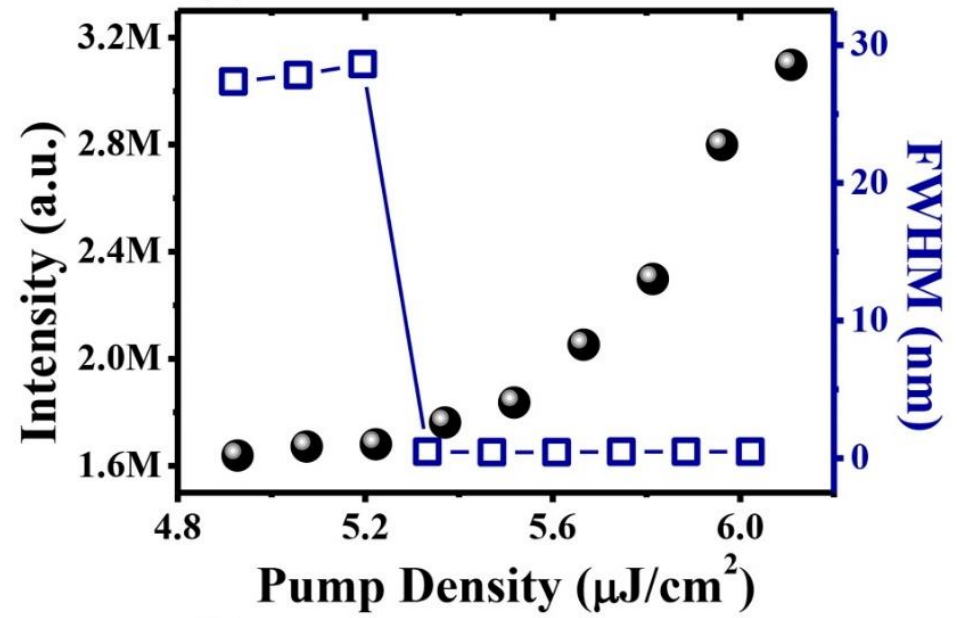

(b)

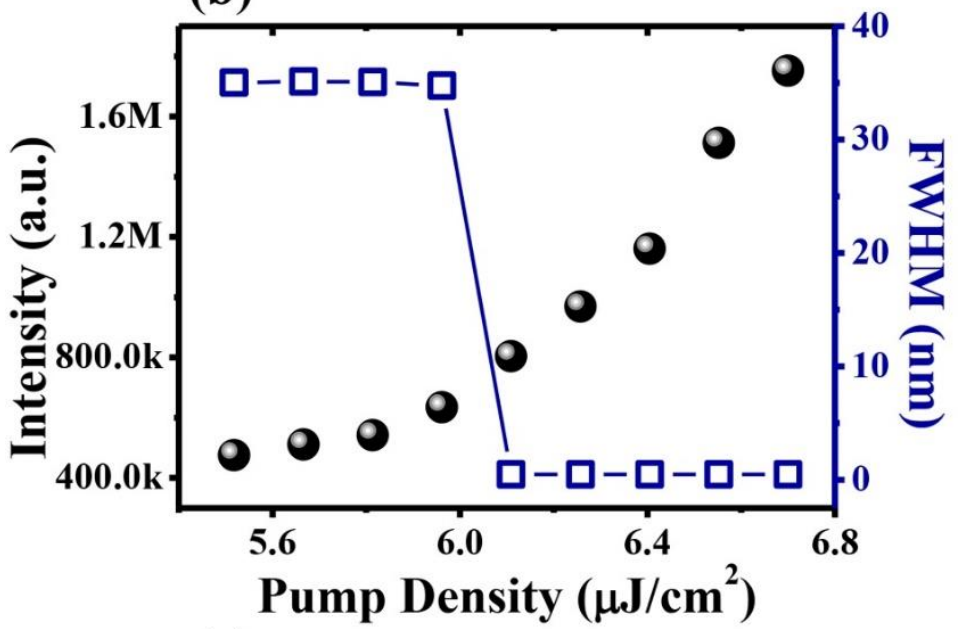

(c)

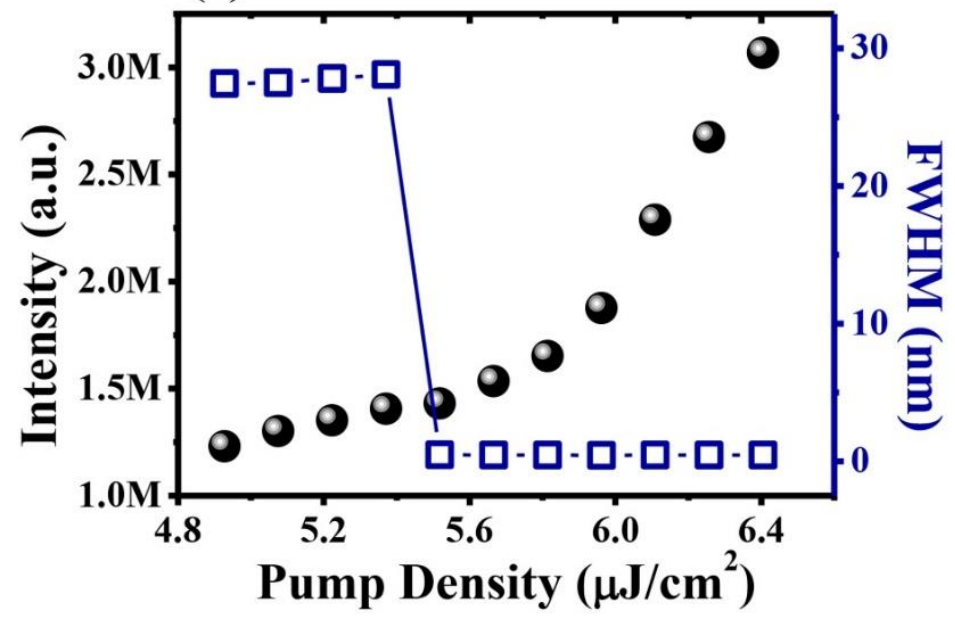


Figure S10. The threshold and full width at half maximum (FWHM) curves of pumped covered half of microplate, the exposed half of microplate and the full pumped microplate.

\section{The fitting of refractive index of $\mathrm{CH}_{3} \mathrm{NH}_{3} \mathrm{PbBr}_{3}$ microplate}

In order to determine the refractive index of the $\mathrm{CH}_{3} \mathrm{NH}_{3} \mathrm{PbBr}_{3}$ perovskite microplate, the measurement of transmission spectrum were carried out. Thus the refractive index at the interference fringe minima and maxima in the transmission spectrum can be calculated by [1]

$$
n=\frac{m \lambda_{m}}{2 d}, \text { (for minima). }
$$

The transmission spectrum of the perovskite microplate is shown in Fig. 11(a). To obtain the value of the refractive index at different wavelength, the Cauchy's equation was employed [2]

$$
n(\lambda)=\mathrm{A}+\frac{B}{\lambda^{2}}+\frac{C}{\lambda^{4}}
$$

By substituting the obtained refractive indices from equation (1) into equation (2), a group of unique solutions of the coefficients $\mathrm{A}, \mathrm{B}$, and $\mathrm{C}$ can be solved. Then the refractive index can be expressed as a function of wavelength. It should be noted that only the refractive index at wavelength larger than the band edge can be fitted with equation (2). The results is summarized in Figure S11(b). In addition, following above analysis process, the refractive index at $550 \mathrm{~nm}$ as a function of reaction time in ICP is presented in Figure S11(c). The refractive index is linearly proportional to the reaction time, indicating the potential tenability of perovskite photoluminescence properties.
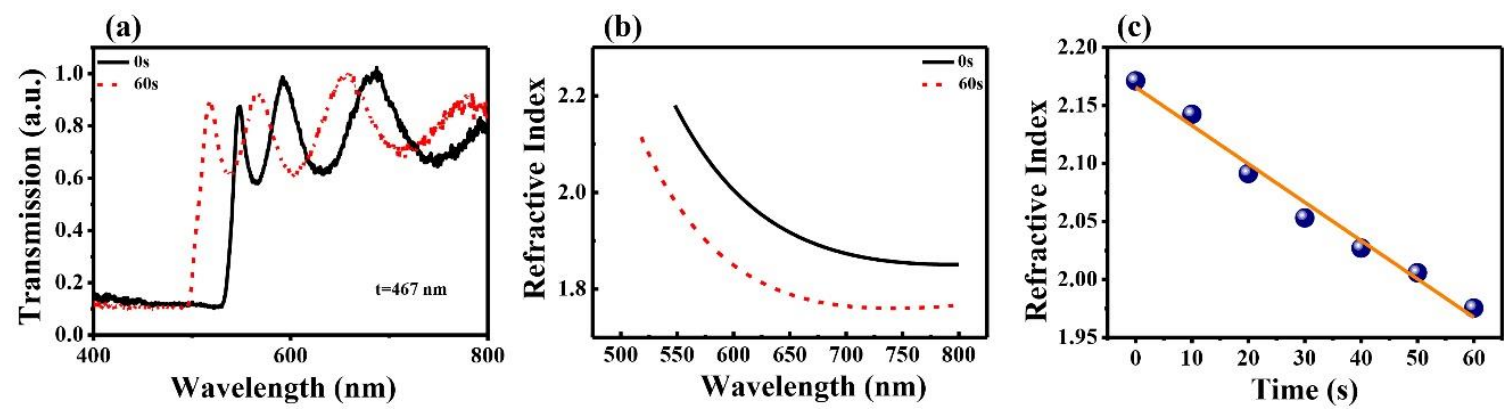
Figure S11. (a) The transmission spectra of microplate, where black solid line is unprocessed spectrum and red dashed line for reaction in ICP for 60s. (b) The fitted refractive index as a function of wavelength abstracted from (a). (c) is the fitted refractive index as a function of reaction time.

\section{Synthesis of perovskite microstructures}

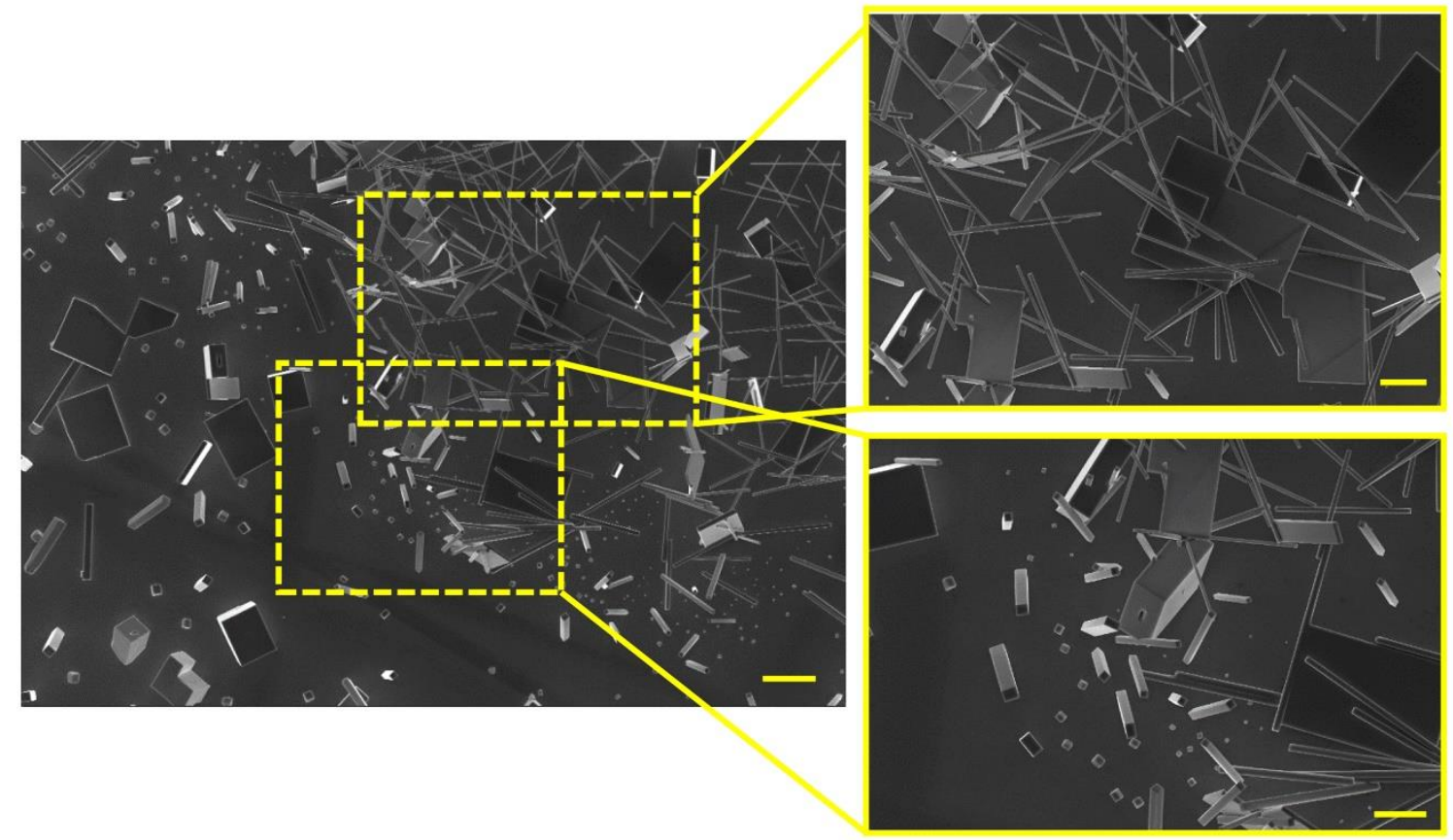

Figure S12. The SEM images of the synthesized microstructures, where the scale bar in left one is $20 \mu \mathrm{m}$ and $10 \mu \mathrm{m}$ for the right ones. The right images are the enlarged images of the left image marked by yellow dashed boxes.

\section{Reference}

[1] Hu, W.; Liu, Z.; Sun, J.; Zhu, S.; Xu, Q.; Feng, D.; Ji. Z. J. Phys. Chem. Solids, 1996, $58,853-857$.

[2] Brunner, D.; Angerer, H.; Bustarret, E.; Freudenberg, F.; Höpler, R.; Dimitrov, R.; Ambacher, O.; Stutzmann. M. J. Appl. Phys. 1997, 82, 5090-5096. The induced coupled plasma (ICP) we have been used is Oxford instruments plasma technology 380 plasma 